\title{
Long non-coding RNA L13Rik promotes high glucose-induced mesangial cell hypertrophy and matrix protein expression by regulating miR- 2861/CDKN1B axis
}

\section{Linlin Sun ( $\nabla$ llsunzj@sina.com )}

Tongren Hospital Shanghai Jiaotong University School of Medicine https://orcid.org/0000-0003-1945$294 X$

\section{Miao Ding}

Tongren Hospital Shanghai Jiaotong University School of Medicine

\section{Fuhua Chen}

Tongren Hospital Shanghai Jiaotong University School of Medicine

\section{Dingyu Zhu}

Tongren Hospital Shanghai Jiaotong University School of Medicine Xinmiao Xie

Tongren Hospital Shanghai Jiaotong University School of Medicine

\section{Xiaoxia Wang}

Tongren Hospital Shanghai Jiaotong University School of Medicine

\section{Research Article}

Keywords: Diabetic nephropathy, Hypertrophy, L13Rik, ceRNA, miR-2861, CDKN1B

Posted Date: February 21st, 2022

DOI: https://doi.org/10.21203/rs.3.rs-1357003/v1

License: (9) This work is licensed under a Creative Commons Attribution 4.0 International License. Read Full License 
Long non-coding RNA L13Rik promotes high glucose-induced mesangial cell hypertrophy and matrix protein expression by regulating miR-2861/CDKN1B axis

L13Rik promotes mesangial cell hypertrophy by regulating miR-2861/CDKN1B axis

Linlin Sun* Miao Ding Fuhua Chen Dingyu Zhu Xinmiao Xie Xiaoxia Wang*

Department of Nephrology

Tongren Hospital, Shanghai Jiao Tong University School of Medicine

1111 Xianxia Road, Shanghai, China, 200336

*Corresponding author: Dr Xiaoxia Wang and Dr Linlin Sun

Dr Linlin Sun:

Email: Ilsunzj@sina.com

Tel 86-18017337095

Fax 021-52039999

Dr Xiaoxia Wang:

Email:WXX2188@shtrhospital.com

Tel 86-18121226865

Fax 021-52039999

Keywords: Diabetic nephropathy, Hypertrophy, L13Rik, ceRNA, miR-2861, CDKN1B 


\section{Abstract}

Glomerular mesangial cell hypertrophy is one of the earliest pathological abnormalities of diabetic nephropathy (DN). A growing literature suggests that lncRNA is involved in the development of DN. However, the function of lncRNA in mesangial cell hypertrophy is still unclear. In the current study, we identified that lncRNA L13Rik was upregulated in DN patients, diabetic rat model and high glucose (HG)-induced mesangial cell. CCK8, Tunel, and western-blot assay were performed to verify the function of silenced L13Rik on the cell growth, apoptosis, and cell hypertrophy in HG-induced mesangial cells. L13Rik knockdown inhibited HG-induced cell proliferation and hypertrophy, while accelerating HG-induced cell apoptosis. Mechanismly, L13Rik regulated mesangial cell hypertrophy by sponging miR-2861. The effect of L13Rik on mesangial hypertrophy was blocked by miR-2861. In addition, L13Rik/miR-2861 promoted mesangial cell hypertrophy by regulating Cyclin-dependent kinase inhibitor 1B (CDKN1B) expression. Taken together, our data showed that L13Rik, as a sponge for miR-2861, regulated protein translation of CDKN1B and led to the hypertrophy of mesangial cell. 


\section{Introduction}

Renal hypertrophy is one of the pathophysiology changes of diabetic nephropathy (DN). The initial stage of $\mathrm{DN}$ is glomerular and mesangial cell hypertrophy, followed by the accumulation of extracellular matrix (ECM) [1, 2]. The kidney is mainly composed of three types of cells: mesangial cell, podocyte, and endothelial cell [3]. Mesangial cell is considered to be the main source of extracellular matrix [4, 5]. P27Kip1-ko-mice failed to develop mesangial hypertrophy from progressive glomerular injury in diabetes [6]. Falguni Das et al. found that the activation of mTOR induced mesangial cell hypertrophy and matrix expansion during early progression of DN by regulating EZH2 [7]. These studies suggested that mesangial cells played a critical role in the progress of DN. High glucose (HG) is one of the main factors of DN that induce mesangial cell hypertrophy. However, the underlying mechanism of HG-induced mesangial cell hypertrophy is still unclear.

Long non-coding RNA (lncRNA) was once regarded as "transcriptional noise," because it did not encode the protein [8]. Until 2007, Rinn et al. confirmed that lncRNA-HOTAIR can inhibit the transcription of HOX gene [9]. To date, the functions of lncRNA are diverse and not fully understood, including antisense interference, cis-regulation, imprinting and ceRNA [10, 11]. Growing evidence suggests that lncRNA plays an important role in the development of DN [12]. Long noncoding RNA Tug1 regulated mitochondrial bioenergetics in DN by enhancing Ppargcla promoter activity [13]. Kato et al. reported that lnc-MGC increased in the glomeruli of mouse models of DN, and targeting lnc-MGC, inhibited glomerular 
ECM and hypertrophy in diabetic mice [14]. However, the lncRNA in the development of HG-induced mesangial hypertrophy is still unclear.

The cell-cycle arrest is a hallmark of mesangial cell hypertrophy [15]. Cyclin-dependent kinase inhibitor 1A (CDKN1A), a kind of Cyclin-dependent kinase inhibitor, is required for glomerular hypertrophy in experimental DN [16]. In addition, CDKN1A or Cyclin-dependent kinase inhibitor 2B (CDKN2B) knock-out mice did not show HG-induced hypertrophy. These studies indicated that CDK inhibitors played a key role in mesangial cell hypertrophy. However, the underlying mechanism of CDK inhibitors regulated by high glucose is still unclear. In the current study, we identified that lncRNA L13Rik was upregulated in DN patients, diabetic rat model and HG-induced mesangial cell, and L13Rik mediated Cyclin-dependent kinase inhibitor 1B (CDKN1B) expression by sponging miR-2861.

\section{Materials and Methods}

\section{Patients and samples}

Human specimens sampling was approved by the Ethics Committee of Shanghai Jiao Tong University Affiliated Tong Ren Hospital, Shanghai, PR China and conducted in accordance with Declaration of Helsinki. In total, $13 \mathrm{DN}$ blood samples were obtained from DN patients aged $53 \pm 12$ ( 7 men and 6 women), and 8 control blood samples were obtained from healthy volunteers aged 55 \pm 10 (4 men and 4 women) in Tong Ren Hospital. DN was diagnosed in accordance with previous criteria [17]. Written informed consent was acquired from each donor before sampling. 
These donors had not received any therapies within 3 months before blood sampling.

\section{Animal model}

All animal procedures were approved by the Ethics Committee of Shanghai Jiao Tong University Affiliated Tong Ren Hospital and performed under the ARRIVE guidelines. 10 Male Sprague-Dawley (SD) rats, aged 8 weeks, were obtained from Model Animal Research Center of Nanjing University (Nanjing, China), and housed in thermostatic room $\left(25 \pm 2{ }^{\circ} \mathrm{C}, 12 / 12\right.$ light/dark cycle, $50 \% \pm 10 \%$ relative humidity). Rats were allowed to access chow and water ad libitum. Rats were weighed and randomly divided into control group $(n=5)$ and diabetic group $(n=5)$. Experimental DN was induced on diabetic rats by intraperitoneal injection with streptozotocin $(50 \mathrm{mg} / \mathrm{kg}$; Aladdin, Shanghai, China) dissolved in sodium citrate buffer ( $\mathrm{pH}=4.5$ ). Access to $20 \%$ glucose water was allowed ad libitum for $18 \mathrm{~h}$ to prevent transient hypoglycemia. $72 \mathrm{~h}$ after streptozotocin administration, the blood glucose of each rat was measured. Rats with blood glucose $>16.7 \mathrm{mmol} / \mathrm{L}$ were considered diabetic. During the experimental period, any rat suffering from disease or dying was euthanized by cervical dislocation under deep anesthesia using pentobarbital sodium $(40 \mathrm{mg} / \mathrm{kg})$.

\section{Cell Culture}

Mouse mesangial cells SV40 MES 13 (CRL-1927TM) were purchased from American Type Culture Collection (ATCC, MA, USA), and maintained in DMEM (Gibco, CA, USA) containing 10\% FBS (Sigma-Aldrich, MO, USA), at $37{ }^{\circ} \mathrm{C}$ under $5 \%$ $\mathrm{CO}_{2}$. To explore the function of glucose concentration on L13Rik expression, 
mesangial cells were challenged with $30 \mathrm{mM}$ mannitol (Ma) or glucose at concentrations of $5 \mathrm{mM}(\mathrm{NG}), 15 \mathrm{mM}$, and $30 \mathrm{mM}(\mathrm{HG})$. To explore the function of treatment time on L13Rik expression, mesangial cells were challenged with $30 \mathrm{mM}$ glucose for $12,24,48$, or $72 \mathrm{~h}$. After exposure to glucose, cells were harvested for quantitative real-time PCR (qRT-PCR) and western blot assay.

\section{Overexpression and RNA interference (RNAi)}

The recombinant lentivirus harboring full-length L13Rik (Lv-L13Rik) and negative control lentivirus were purchased from Sangon (Shanghai, China). L13Rik siRNA (siL13Rik) and miR-2861 mimics were synthesized by Genewiz (Jiangsu, China), and transfected into mesangial cells with Lipofectamine RNAiMAX (Invitrogen, CA, USA) according to the manufacturer's introduction. The gene expression after treatment of Lv-L13Rik or siL13Rik was measured by qRT-PCR.

\section{Cell proliferation assay}

The viability of mesangial cells was assayed with Cell Counting Kit-8 (Beyotime, Shanghai, China) following the manufacturer's protocols. Briefly, mesangial cells were resuspended and assigned to HG group and NG group. Then, cells were plated into 96-well plates at a density of $3 \times 10^{5}$ cells per well. HG mesangial cells were cultured in medium containing $30 \mathrm{mM}$ glucose in the presence or absence of miR-2861 mimics. NG mesangial cells were cultured in medium containing $5 \mathrm{mM}$ glucose without siRNA treatment. After culturing at $37{ }^{\circ} \mathrm{C}$ for $24 \mathrm{~h}$, cells were incubated with $10 \mu \mathrm{L}$ of CCK-8 reagent at $37{ }^{\circ} \mathrm{C}$ for $120 \mathrm{~min}$. The absorbance $(450$ $\mathrm{nm}$ ) of each well was measured on a microplate reader (HENGMEI TECHNOLOGY, 
Shandong, China).

\section{Cell apoptosis assay}

NG-treated mesangial cells and HG-treated mesangial cells were seeded in 12-well plates, and HG-treated mesangial cells were cultured in the presence or absence of siL13Rik/miR-2861 mimics treatment. After incubation at $37{ }^{\circ} \mathrm{C}$ for $48 \mathrm{~h}$ and fixation with $4 \%$ paraformaldehyde, TUNEL staining was carried out with One Step TUNEL Apoptosis Assay Kit (Beyotime). Nuclei were labeled using DAPI (Beyotime). The fluorescence signal was captured using MF53-M microscope (Mshot, Guangdong, China).

\section{Fluorescence in Situ Hybridization (FISH)}

The L13Rik probe was synthesized by Sangon (Shanghai, China). After culturing in 12-well plates in the presence or absence of high glucose for $48 \mathrm{~h}$, mesangial cells were washed by PBS and fixed by $4 \%$ PFA for 15 minutes at room temperature, following treatment with proteinase $\mathrm{K}$, glycine and acetic anhydride. Pre-hybridization was performed at $37{ }^{\circ} \mathrm{C}$ for $1 \mathrm{~h}$, followed by hybridization L13Rik against using $250 \mathrm{ng} / \mathrm{mL}$ of L13Rik probe. Nuclei were labeled with DAPI, and L13Rik signal was captured as mentioned earlier.

\section{qRT-PCR}

Total RNA in blood samples and mesangial cells was extracted with RNA simple Total RNA Kit (Tiangen, Beijing, China) according to the manufacturer's introduction. cDNA was synthesized with TIANScript II RT Kit (Tiangen) at $42{ }^{\circ} \mathrm{C}$ for $60 \mathrm{~min}$. qPCR was performed in triplicate with FastFire qPCR PreMix (SYBR Green) 
(Tiangen) on ViPlex Fluor Real-time PCR system (Vivantis, Selangor, Malaysia). Thermocycling conditions were set as follows: $95{ }^{\circ} \mathrm{C}$ for $60 \mathrm{~s}$, followed by 30 cycles of $95{ }^{\circ} \mathrm{C}$ for $15 \mathrm{~s}$ and $67^{\circ} \mathrm{C}$ for $30 \mathrm{~s}$. Gene expressions were calculated by $2^{-\Delta \Delta \mathrm{CT}}$ method. Expressions of Fibronectin (FN), collagen IV (Col-IV), N-cadherin (N-cad), CDKN1B and L13Rik were normalized by GAPDH, and expression of miR-2861 was normalized by U6. All primer sequences were listed in Table-1.

\section{Western blot}

Mesangial cells were lysed with xTractor Buffer Kit (CLONTECH Laboratories, Inc., CA, USA). Protein specimens were quantified through BCA method and electrophoresed through $10 \%$ SDS-PAGE gels. After transferring onto PVDF membranes, interested proteins were masked with $2 \%$ non-fat milk at $37{ }^{\circ} \mathrm{C}$ for 30 min, then incubated with anti-FN antibody (1:700; SAB5700724; Sigma-Aldrich), anti-Col IV antibody (1: 500; ab52235; Abcam, CA, USA), anti-CDKN1B antibody (1: 1000; ab32034; Abcam), anti-N-cad antibody (1:8000; ab76011; Abcam), and anti- $\beta$-actin antibody (1:20000; AF7018; Affinity, Jiangsu, China) at $4{ }^{\circ} \mathrm{C}$ for $20 \mathrm{~h}$. The membranes were rinsed 3 times with PBST, then immersed in HRP-conjugated anti-rabbit IgG secondary antibody solution (1: 5000; S0001; Affinity) at $37{ }^{\circ} \mathrm{C}$ for 60 min. Interesting proteins were visualized using commercial ECL kit (Glpbio, CA, USA). Fluorescence intensities were quantified using Fiji software.

\section{RNA pull-down assay}

Biotin-labeled miR-2861 was purchased from Genewiz (Jiangsu, China). Biotin-labelled miR-2861 (3 $\mu \mathrm{g})$ was incubated with streptavidin-coated beads 
(434341; Thermofisher Scientific) at $4{ }^{\circ} \mathrm{C}$ overnight. Then, streptavidin-coated beads were incubated with mesangial cell lysate at $4{ }^{\circ} \mathrm{C}$ for $12 \mathrm{~h}$. RNAs bound by miR-2861 were purified with Trizol reagent (Takara, Shiga, Japan), and detected through qRT-PCR assay.

\section{RNA immunoprecipitation (RIP) assay}

After miR-2861 knockdown, RIP assay was performed as per the instructions of the Immunoprecipitation Kit with Protein A+G Magnetic Beads (Beyotime). At first, $10^{6}$ miR-2861-knockdown mesangial cells and control mesangial cells were lysed respectively with $200 \mu \mathrm{L}$ RIPA buffer (Beyotime). Lysates were incubated with $20 \mu 1$ of magnetic beads labeled with normal mouse IgG (A7028; Beyotime) or rabbit anti-Ago2 antibody (DF12246; Affinity) at $4{ }^{\circ} \mathrm{C}$ for $16 \mathrm{~h}$. Immunoprecipitated RNA was obtained by digesting protein with Proteinase-K, and quantified on a microplate reader. Lastly, immunoprecipitated L13Rik and miR-2861 were assayed through qRT-PCR.

\section{Dual - luciferase reporter assay}

The wild-type or mutant predictive binding site of miR-2861 on L13Rik was cloned into PGL3 vector (Fenghui Biotechnology, Hunan, China) to construct pGL3-L13Rik-WT or pGL3-L13Rik-Mut plasmids. 3'UTR of CDKN1B or its mutant was inserted into PGL3 vector to construct pGL3-CDKN1B-3'UTR-WT or pGL3-CDKN1B-3'UTR-Mut plasmids. $10^{6}$ HEK-293T cells were briefly plated into 24-well plates. 20ng of pGL3-L13Rik-WT, pGL3-L13Rik-Mut, pGL3-CDKN1B-3'UTR-WT or pGL3-CDKN1B-3'UTR-Mut plasmids was 
co-transfected with $40 \mathrm{nM}$ of miR-2861, and $2 \mathrm{ng}$ of pRL-TK (Beyotime) into HEK-293T cells with Lipofectamine ${ }^{\mathrm{TM}} 3000$ according to the manufacturer's protocol. After incubation at $37{ }^{\circ} \mathrm{C}$ for $48 \mathrm{~h}$, luciferase activities of each well were assayed using Dual-Luciferase Reporter Gene Assay Kit (Beyotime) in accordance with the manufacture's protocol. Renilla luciferase was used for an internal control.

\section{Statistical analysis}

Dual-luciferase reporter assay, qRT-PCR, western bolt and CCK-8 assay were performed in triplicate. Quantified data was analyzed using SPSS 22.0 software (IBM, USA) and presented as MEAN \pm standard error of the mean (SEM). Comparisons between groups were performed through student's $t$-test or one-way analysis of variance. P-value less than 0.05 was defined as statistically significant.

\section{Results}

\section{L13Rik upregulated in DN patients, diabetic rats, and HG-treated mesangial} cells.

Transforming growth factor-beta (TGF-beta) is closely associated with progressive renal hypertrophy, fibrosis, and is applied to glomerular mesangial cell models of extracellular matrix (ECM) accumulation. In the current study, we found L13Rik was upregulated in the TGF-beta-treated mesangial cells and got its full length from the noncode database (http://www.noncode.org/index.php) (supporting Table1). However, the function of L13Rik in renal hypertrophy and fibrosis is still unclear. To explore the possible effect of L13Rik in HG-induced mesangial cell hypertrophy, we first measured L13Rik expression in 13 DN patients and 8 healthy 
controls who were recruited. As shown in Figure 1A, L13Rik expression was markedly upregulated in peripheral blood of DN patients compared with the healthy controls. Next, the expression of L13Rik was further confirmed in diabetic rats. Similarly, L13Rik expression was significantly increased in renal tissues of diabetic rats compared with control rats (Figure 1B). Consistently, Figure 1C shows that L13Rik expression was significantly upregulated in HG-cultured mesangial cells. The relationships between treatment time and glucose concentration on L13Rik expression were also explored. As shown in Figure 1D and E, the expression of L13Rik was obviously increased in a time-dependent (Figure 1D) and dose-dependent (Figure 1E) manner. Furthermore, FISH results also verified that L13Rik was upregulated in HG-cultured mesangial cells (Figure 1F). We also found that L13Rik was mainly located in the cytoplasm of mesangial cells. Taken together, our results indicated that L13Rik may contribute to the development of DN through regulating mesangial cell hypertrophy.

\section{Knockdown of L13Rik suppressed HG-induced proliferation, ECM accumulation and hypertrophy}

To investigate the effect of L13Rik in DN, L13Rik knockdown or overexpression was performed to investigate its functions. CCK8 assay was performed to confirm the cell viability of mesangial cells. Figure $2 \mathrm{~A}$ shows that the cell viability of mesangial cells was markedly increased in the HG group, while the effect was blocked by siL13Rik. Tunel assay showed that the cell apoptosis of mesangial cells was markedly decreased in the HG group, while the effect was restored by siL13Rik (Figure 2B). 
To identify the effect of L13Rik on ECM accumulation in mesangial cells, the expressions of FN, Col-IV and N-cad were assessed by RT-PCR and western blot. Figure 2C-E shows that the mRNAs and protein levels of FN, Col-IV and N-cad were markedly increased in the HG group, while the effect was blocked by siL13Rik. Similar data was found in the mesangial cell hypertrophy, our data showed that the upregulation of total protein level per cell induced by HG was significantly inhibited by siL13Rik (Figure 2F).

\section{L13Rik sponged miR-2861 by acting as ceRNA}

CeRNA is one of the classical regulatory mechanisms for cytoplasmic location IncRNA. Thus, 63 candidate miRNAs sponged by L13Rik were predicted by bioinformatics (http://mirdb.org/cgi-bin/search.cgi.) (supplementary Table2). 86 dysregulated miRNAs were reported in human DN studies (supplementary Table3). Venn analysis confirmed that miR-2861 was the only miRNA that could be sponged by L13Rik and dysregulated in DN patients (supplementary Figure S2).

MiR-2861 expression was assessed in 13 DN patients and 8 healthy controls. As expected, miR-2861 expression was obviously decreased in peripheral blood of DN patients compared with healthy controls (Figure 3A). Similarly, Figure 3B shows that miR-2861 expression was significantly downregulated in HG-cultured mesangial cells. In addition, miR-2861 expression was markedly decreased in a dose-dependent (Figure 3C) manner. The binding sites between L13Rik and miR-2861 were predicted using bioinformatics analysis. To explore the direct binding of L13Rik to miR-2861, pGL3-L13Rik-WT or pGL3-L13Rik-Mut was constructed and co-transfected with 
miR-2861(Figure 3D). As shown in Figure 3D, miR-2861 significantly decreased the fluorescence of wide type L13Rik but had no impact on mutant L13Rik. Consistently, miR-2861 mutant lost the inhibitor effect to luciferase activity of pGL3-L13Rik-WT (Figure 3E). The direct interaction of miR-2861 with L13Rik was further investigated by RNA pull-down assay with biotin-labelled miR-2861. As shown in Figure 3F, a significant enrichment of L13Rik was observed in biotin-labelled miR-2861 group compared with biotin-labelled miRcont. RIP assay using anti-Ago2 antibody showed that L13Rik and miR-2861 were enriched in control cells, while miR-2861 knockdown obviously decreased the enrichment of L13Rik and miR-2861 in Ago2 complexes (Figure $3 \mathrm{G}$ and $\mathrm{H})$.

MiR-2861 suppressed HG-induced proliferation, ECM accumulation and hypertrophy

The correlation of miR-2861 with mesangial cell proliferation, ECM accumulation and hypertrophy was investigated. The cell viability of mesangial cells with or without HG in the absence or presence of miR-2861 was assessed by CCK-8. As shown in Figure 4A, miR-2861 significantly inhibited HG-induced mesangial cell proliferation. The cell apoptosis of mesangial cells with or without HG in the absence or presence of miR-2861 was analyzed by Tunel. As shown in Figure 4B, the mesangial cell apoptosis induced by HG was blocked by miR-2861. The expressions of ECM accumulation-related genes FN, Col-IV, and N-cad were assessed by qRT-PCR and western blot. Figure 4 C-E show that the upregulations of FN, Col-IV and N-cad induced by HG were suppressed by miR-2861. Similarly, the upregulation 
of the total protein level per cell induced by HG was blocked by miR-2861(Figure 4F). Taken together, these data indicated that miR-2861 suppressed HG-induced proliferation, ECM accumulation and hypertrophy.

\section{MiR-2861 antagonized the effect of L13Rik on hypertrophy}

To explore the role of L13Rik/miR-2861 in mesangial cells, mesangial cells were co-transfected with the miR-2861 mimic and the L13Rik-overexpressing lentivirus. As shown in Figure 5A, L13Rik promoted cell proliferation, while the effect was blocked by miR-2861. Moreover, L13Rik increased the expressions of FN, Col-IV, and N-cad, whereas miR-2861 mimic reversed the effects (Figure 5B-D). The cell hypertrophy data showed that L13Rik increased the total protein level per cell, while the effect was blocked by miR-2861 mimic (Figure 5D). In conclusion, our data indicated that L13Rik promoted mesangial cell proliferation, ECM accumulation and hypertrophy by sponging miR-2861.

\section{L13Rik promoted cell hypertrophy by modulating miR-2861/CDKN1B axis}

The potential target genes of miR-2861 were predicted by the database TargetScan (http://www.targetscan.org/vert_71/). Total 4407 genes were predicted possibly targeted by miR-2861. Among these genes, CDKN1B was previously reported to be associated with mesangial cell hypertrophy [6]. To confirm the statement, plasmids pGL3-CDKN1B-3'UTR-WT or pGL3-CDKN1B-3'UTR-Mut were constructed and co-transfected with miR-2861 (Figure 6A). As shown in Figure 6B, miR-2861 significantly decreased the fluorescence of wide type CDKN1B but had no impact on mutant CDKN1B. Moreover, CDKN1B expression was further 
assessed by western blot. Figure 6C and D show that CDKN1B expression was obviously decreased by miR-2861. Given that L13Rik sponged miR-2861 and CDKN1B is the miR-2861 target gene, we concluded that L13Rik might regulate CDKN1B expression by sponging miR-2861. Figure 6E and F show that L13Rik increased CDKN1B expression, while the effect was restored by miR-2861.

\section{Discussion}

Renal hypertrophy, especially glomerular hypertrophy, plays a core role in the pathology of DN [18]. LncRNA was reported to be involved in the progress of DN [12]. However, the function of lncRNA in renal hypertrophy is still unclear. In the current study, we demonstrated that long non-coding RNA L13Rik promoted HG-induced matrix protein expression and mesangial cell hypertrophy by regulating miR-2861/ CDKN1B axis, as evidenced by the following: 1) Upregulated L13Rik was found in DN patients, diabetic rats and HG-treated mesangial cells; 2) Knockdown of L13Rik suppressed HG-induced proliferation, ECM accumulation and hypertrophy; 3) L13Rik sponged miR-2861 by acting as ceRNA; 4) MiR-2861 suppressed HG-induced proliferation, ECM accumulation and hypertrophy; 5) MiR-2861 antagonized the effect of L13Rik on hypertrophy; 6) L13Rik promoted cell hypertrophy by modulating miR-2861/CDKN1B axis.

Growing studies have suggested that lncRNA was involved in the progress of DN. Huang et al. demonstrated that MALAT1 accelerated kidney fibrosis by regulating miR-2355-3p/IL4ST axis [19]. Duan et al. reported that lnc-ISG20 promoted kidney fibrosis in DN by regulating miR-486-5p/NFAT5-induced AKT 
phosphorylation [20]. LncRNA location in the cytoplasm can regulate the transcription of mRNA by sponging miRNAs. In our study, we showed that lncRNA L13Rik was increased in DN patients, diabetic rats and HG-induced mesangial cells, and L13Rik was confirmed to be located in the cytoplasm of mesangial cells. Next, the bioinformatics prediction, dual-RNA pull-down, RIPA assay and luciferase-reporter gene assay were performed to verify that L13Rik directly sponge miR-2861 and negatively regulates miR-2861 activity.

A previous study found that miR-2861 was decreased in DN patients, and was associated with estimated glomerular filtration rate [21]. However, the mechanism of miR-2861 in the development of DN is still unclear. As expected, our results showed that miR-2861 was decreased in DN patients and HG-induced mesangial cells. Furthermore, we found that the mesangial cell hypertrophy induced by $\mathrm{HG}$ and L13Rik was blocked by miR-2861, suggesting that miR-2861 mediated the function of L13Rik in the progress of DN and mesangial cell hypertrophy.

Cyclin-dependent kinase inhibitor 1B (CDKN1B, p27Kip1) was reported to be involved in renal hypertrophy $[6,22] . \mathrm{G}$ Wolf et al. reported that HG promoted p27Kip1 expression in cultured mouse mesangial cells and may concubine to the process of diabetic glomerular hypertrophy [23]. Liao et al. demonstrated that NEAT1 accelerated HG-induced mesangial cell hypertrophy by regulating miR-222-3p/CDKN1B axis [24]. In the current study, we found that CDKN1B was the direct target of miR-2861, as evidenced by the bioinformatics prediction, dual-luciferase-reporter gene and western blotting assay. In addition, we also 
confirmed that L13Rik promoted CDKN1B expression by sponging miR-2861. In conclusion, our study clarifies that lncRNA L13Rik promotes high glucose-induced mesangial cell hypertrophy and matrix protein expression by regulating mir-2861/ CDKN1B axis.

\section{Compliance with Ethical Standards}

\section{Conflict of interest}

On behalf of all authors, the corresponding author states that there is no conflict of interest.

\section{Ethical approval}

All procedures performed in this study were in accordance with the ethical standards of the institutional and international research committee and with the 1964 Helsinki declaration and its later amendments or comparable ethical standards.

\section{Informed consent}

Informed consent was obtained from all participants included in the study.

\section{Funding Sources}

This study was funded by Natural Science Foundation of Shanghai (grant number 20ZR1451600), Shanghai Municipal Health Bureau Project (grant number 201940439).

\section{Reference:}

1. Rojas-Canales DM, Li JY, Makuei L, et al (2019) Compensatory renal hypertrophy following nephrectomy: When and how? Nephrology (Carlton) 24(12): 1225-1232.

2. Sun HJ (2019) Current Opinion for Hypertension in Renal Fibrosis. Adv Exp Med Biol 1165: 37-47.

3. Wallace MA (1998) Anatomy and physiology of the kidney. AORN J 68(5): 800, 803-16, 819-20; quiz 821-4.

4. Tung CW, Hsu YC, Shih YH, et al (2018) Glomerular mesangial cell and podocyte injuries in diabetic nephropathy. Nephrology (Carlton) 23 Suppl 4: 32-37. 
5. $\quad$ Abboud HE (2012) Mesangial cell biology. Exp Cell Res 318(9): 979-85.

6. Awazu M, Omori S, Ishikura K, et al (2003) The lack of cyclin kinase inhibitor p27(Kip1) ameliorates progression of diabetic nephropathy. J Am Soc Nephrol 14(3): 699-708.

7. Das F, Bera A, Ghosh-Choudhury N, et al (2021) High glucose-stimulated enhancer of zeste homolog-2 (EZH2) forces suppression of deptor to cause glomerular mesangial cell pathology. Cell Signal 86: 110072.

8. Jiang ZF, Zhang L (2021) LncRNA: A Potential Research Direction in Intestinal Barrier Function. Dig Dis Sci 66(5): 1400-1408.

9. Rinn JL, Kertesz M, Wang JK, et al (2007) Functional demarcation of active and silent chromatin domains in human HOX loci by noncoding RNAs. Cell 129(7): 1311-23.

10. Cech TR, Steitz JA (2014) The noncoding RNA revolution-trashing old rules to forge new ones. Cell, 157(1): 77-94.

11. Holoch D, Moazed D (2015) RNA-mediated epigenetic regulation of gene expression. Nat Rev Genet, 16(2): 71-84.

12. Coellar JD, Long J, Danesh FR (2021) Long Noncoding RNAs and Their Therapeutic Promise in Diabetic Nephropathy. Nephron 145(4): 404-414.

13. Long J, Badal SS, Ye Z, et al (2016) Long noncoding RNA Tug1 regulates mitochondrial bioenergetics in diabetic nephropathy. J Clin Invest 126(11): 4205-4218.

14. Kato $M$, Wang $M$, Chen $Z$, et al (2016) An endoplasmic reticulum stress-regulated IncRNA hosting a microRNA megacluster induces early features of diabetic nephropathy. Nat Commun 7: 12864.

15. Shankland, S.J (1999) Cell cycle regulatory proteins in glomerular disease. Kidney Int 56(4): 1208-1215.

16. Al-Douahji M, Brugarolas J, Brown PA, et al (1999) The cyclin kinase inhibitor p21WAF1/CIP1 is required for glomerular hypertrophy in experimental diabetic nephropathy. Kidney Int, 56(5): 1691-1699.

17. An Y, Xu F, Le W, et al (2015) Renal histologic changes and the outcome in patients with diabetic nephropathy. Nephrol Dial Transplant 30(2): 257-266.

18. Dai H, Liu Q, Liu B (2017) Research Progress on Mechanism of Podocyte Depletion in Diabetic Nephropathy. J Diabetes Res 2017: 2615286.

19. Huang H, Zhang G, Ge Z (2021) IncRNA MALAT1 Promotes Renal Fibrosis in Diabetic Nephropathy by Targeting the miR-2355-3p/IL6ST Axis. Front Pharmacol 12: 647650.

20. Duan YR, Chen BP, Chen F, et al (2021) LncRNA Inc-ISG20 promotes renal fibrosis in diabetic nephropathy by inducing AKT phosphorylation through miR-486-5p/NFAT5. J Cell Mol Med, 25(11): 4922-4937.

21. Cardenas-Gonzalez M, Srivastava A, Pavkovic M, et al (2017) Identification, Confirmation, and Replication of Novel Urinary MicroRNA Biomarkers in Lupus Nephritis and Diabetic Nephropathy. Clin Chem 63(9): 1515-1526.

22. Wolf G, Jablonski K, Schroeder R, et al (2003) Angiotensin Il-induced hypertrophy of proximal tubular cells requires p27Kip1. Kidney Int 64(1): 71-81.

23. Wolf G, Schroeder R, Ziyadeh FN, et al (1997) High glucose stimulates expression of p27Kip1 in cultured mouse mesangial cells: relationship to hypertrophy. Am J Physiol 273(3 Pt 2): F348-356.

24. Liao L, Chen J, Zhang C, et al (2021) LncRNA NEAT1 Promotes High Glucose-Induced 
Mesangial Cell Hypertrophy by Targeting miR-222-3p/CDKN1B Axis. Front Mol Biosci 7: 627827.

\section{Figure legends}

Figure 1. L13Rik upregulated in DN patients, diabetic rats, and HG-treated mesangial cells.

(A-C) qPCR analysis of L13Rik expression in 13 DN patients and 8 healthy controls (A), in renal tissues of diabetic rats (B), and in HG-cultured mesangial cells (C). (D and E) The level of L13Rik in various conditions as indicated was assessed by qPCR. The results from qRT-PCR showed that L13Rik was increased in a time (D) and dose-dependent manner (E). (F) FISH analysis for L13Rik sub-cellularlocalization in mesangial cells. Probes targeting L13Rik were stained in green and the nucleuses were stained in blue. ${ }^{*} p<0.05 . * * p<0.01$.

Figure 2. Knockdown of L13Rik suppressed HG-induced proliferation, ECM accumulation, and hypertrophy

(A) Mesangial cell proliferation was assayed using CCK-8 after HG treatment with or without L13Rik knockdown. (B) Mesangial cell apoptosis was assayed using Tunel staining after HG treatment with or without L13Rik knockdown. (C) The mRNA levels of fibronectin (FN), collagen IV (Col-IV) and N-cadherin (N-cad) in mesangial cells after HG treatment with or without L13Rik knockdown were assessed by RT-PCR. (D and E) The protein levels of FN, Col-IV and N-cad in mesangial cells after HG treatment with or without L13Rik knockdown were assessed by western blotting. (F) The mesangial cell hypertrophy was assessed using total protein level per cell. The results show data from three independent experiments, expressed as the mean \pm SD. ${ }^{*} p<0.05$. $* * p<0.01$ 
Figure 3. L13Rik sponged miR-2861 by acting as ceRNA

(A-C) qRT-PCR analysis of miR-2861 expression in 13 DN patients and 8 healthy controls (A), in diabetic rats (B), and in HG-cultured mesangial cells (C). (D) Schematic diagram of the miR-2861 site in L13Rik-wt-3'UTR and L13Rik-mutant-3'UTR. Luciferase activity was assayed in HEK293T cells co-transfected with miR-2861 and luciferase reporters containing L13Rik-wt-3'UTR and L13Rik-mutant-3'UTR. Data is presented as the relative ratio of firefly luciferase activity to renilla luciferase activity. (E) Luciferase activity was assayed in HEK293T cells co-transfected with L13Rik-wt-3'UTR with miR-2861-wt or miR-2861-Mut. Data is presented as the relative ratio of firefly luciferase activity to renilla luciferase activity. (F) The direct interaction of miR-2861 with L13Rik was assessed by RNA pull-down assay. (G-H) RNA immunoprecipitation (RIP) analyses with anti-Ago2 antibody were carried out to assess the enrichment of L13Rik and miR-2861. The results show data from three independent experiments, expressed as the mean \pm SD. ${ }^{*} p<0.05 . * * p<0.01$.

Figure 4. MiR-2861 suppressed HG-induced proliferation, ECM accumulation and hypertrophy

(A) Mesangial cell proliferation was assayed using CCK-8 after HG treatment with or without miR-2861 overexpression. (B) Mesangial cell apoptosis was assayed using Tunel staining after HG treatment with or without miR-2861 overexpression. (C) The mRNA levels of FN, Col-IV and $\mathrm{N}$-cad in mesangial cells after HG treatment with or without miR-2861 overexpression were assessed by RT-PCR. (D and E) The protein levels of FN, Col-IV and N-cad in mesangial cells after HG treatment with or without miR-2861 overexpression were assessed by western blotting. 
(F) The mesangial cell hypertrophy after HG treatment with or without miR-2861 overexpression was assessed using total protein level per cell. The results show data from three independent experiments, expressed as the mean $\pm \mathrm{SD} . * p<0.05$. ${ }^{* *} p<0.01$.

\section{Figure 5. MiR-2861 antagonized the effect of L13Rik on hypertrophy}

(A) Mesangial cell proliferation was assayed using CCK-8 after overexpression with L13Rik in the presence or absence of miR-2861. (B) The mRNA levels of FN, Col-IV, and N-cad in mesangial cells after overexpression with L13Rik in the presence or absence of miR-2861 were assessed by RT-PCR. (C and D) The protein levels of FN, Col-IV and N-cad in mesangial cells after overexpression with L13Rik in the presence or absence of miR-2861were assessed by western blotting. The results show data from three independent experiments, expressed as the mean \pm SD. $* * p<0.01$.

\section{Figure 6. L13Rik promoted cell hypertrophy by modulating miR-2861/CDKN1B axis}

(A) Schematic diagram of the miR-2861 site in CDKN1B-3'UTR. (B) The activation of Luciferase was analyzed in HEK293T cells co-transfected with miR-2861 and luciferase reporters containing CDKN1B-3'UTR-Wt or CDKN1B-3'UTR-Mut. (C and D) Western blot analysis of CDKN1B expression in mesangial cells after miR-2861 overexpression. (E and F) Western blot analysis for CDKN1B protein level in mesangial cells after L13Rik overexpression in the presence or absence of miR-2861. The results show data from three independent experiments, expressed as the mean \pm SD. ${ }^{*} p<0.05 . * * p<0.01$. 
A

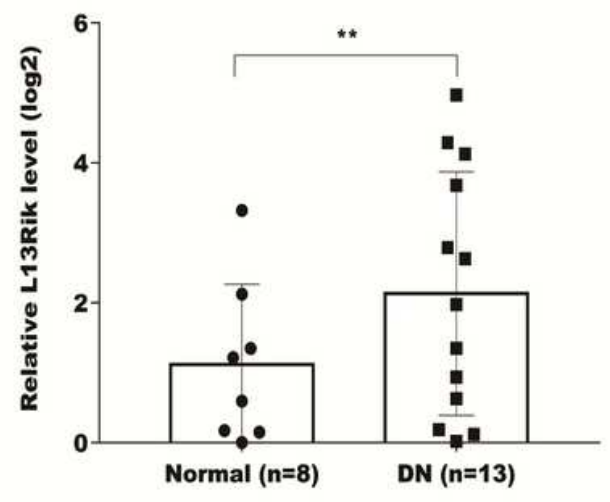

D

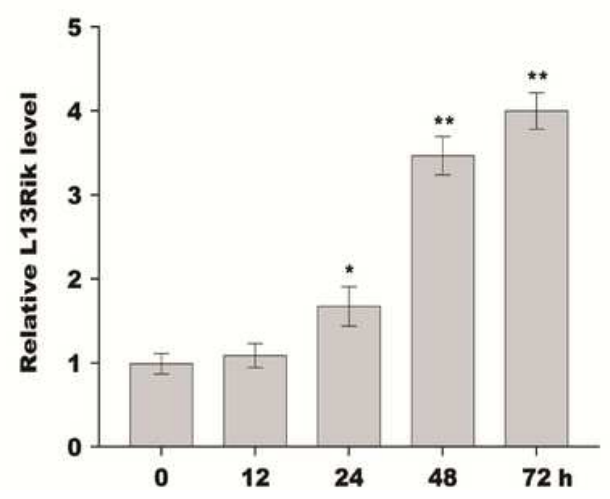

B

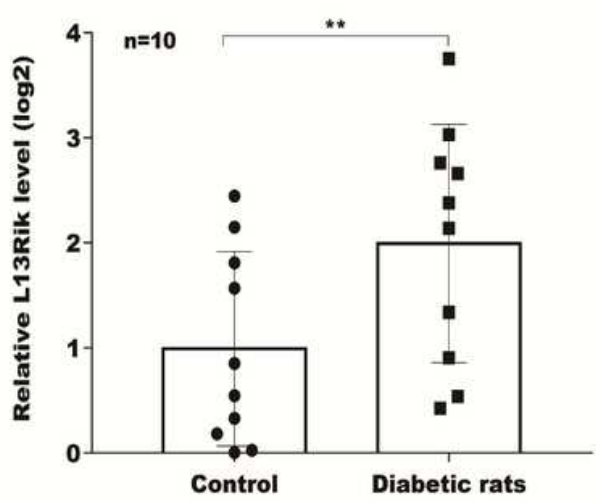

E

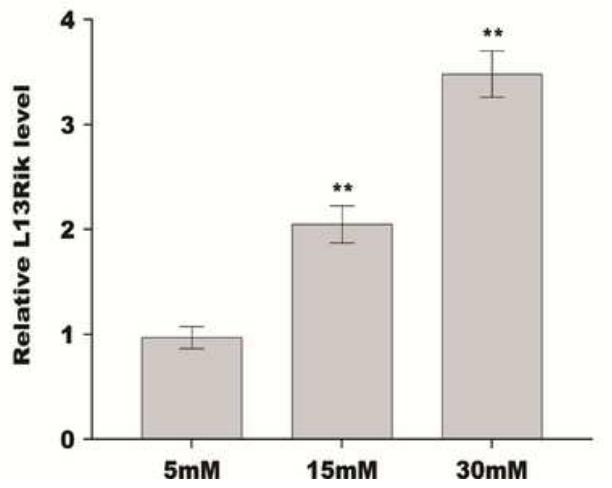

C

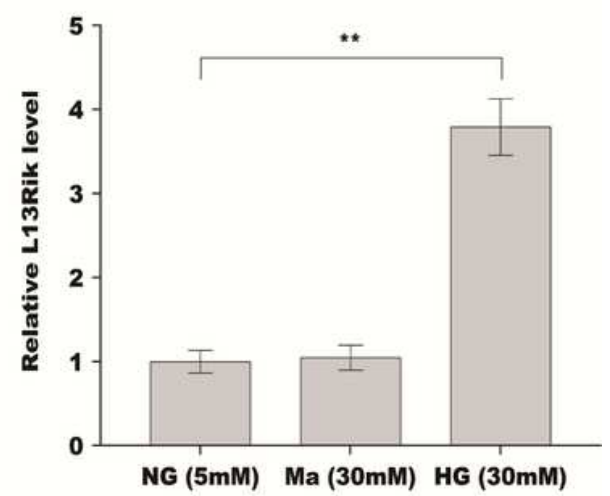

$\mathbf{F}$

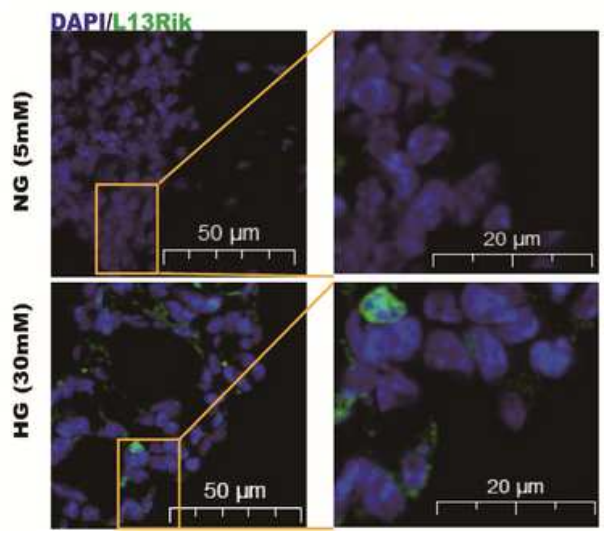

Figure 1

L13Rik upregulated in DN patients, diabetic rats, and HG-treated mesangial cells. (A-C) qPCR analysis of L13Rik expression in 13 DN patients and 8 healthy controls (A), in renal tissues of diabetic rats (B), and in HG-cultured mesangial cells (C). (D and E) The level of L13Rik in various conditions as indicated was assessed by qPCR. The results from qRT-PCR showed that L13Rik was increased in a time (D) and dosedependent manner (E). (F) FISH analysis for L13Rik sub-cellularlocalization in mesangial cells. Probes targeting L13Rik were stained in green and the nucleuses were stained in blue. ${ }^{*} p<0.05 .{ }^{* *} p<0.01$. 
A

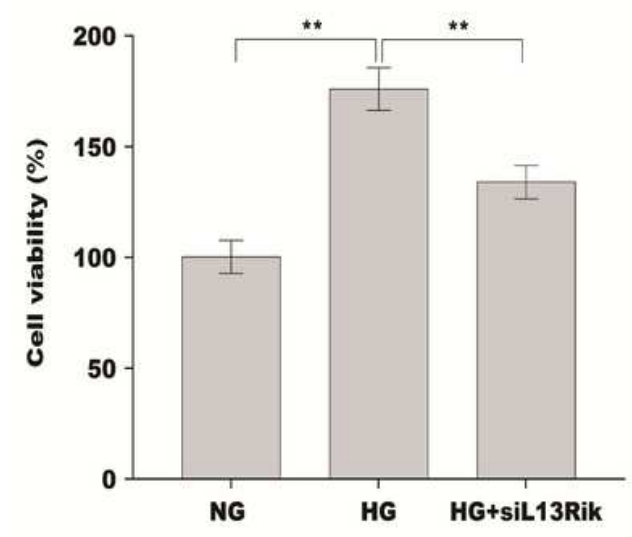

C

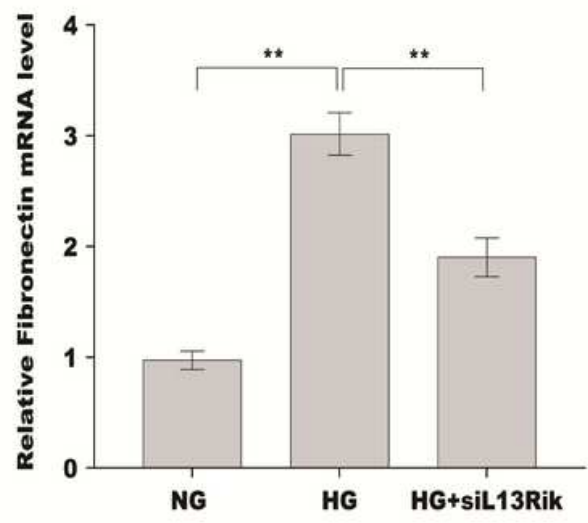

D

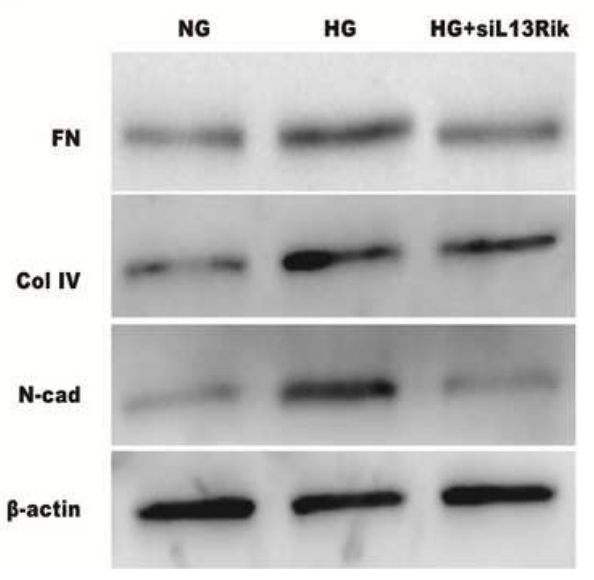

B
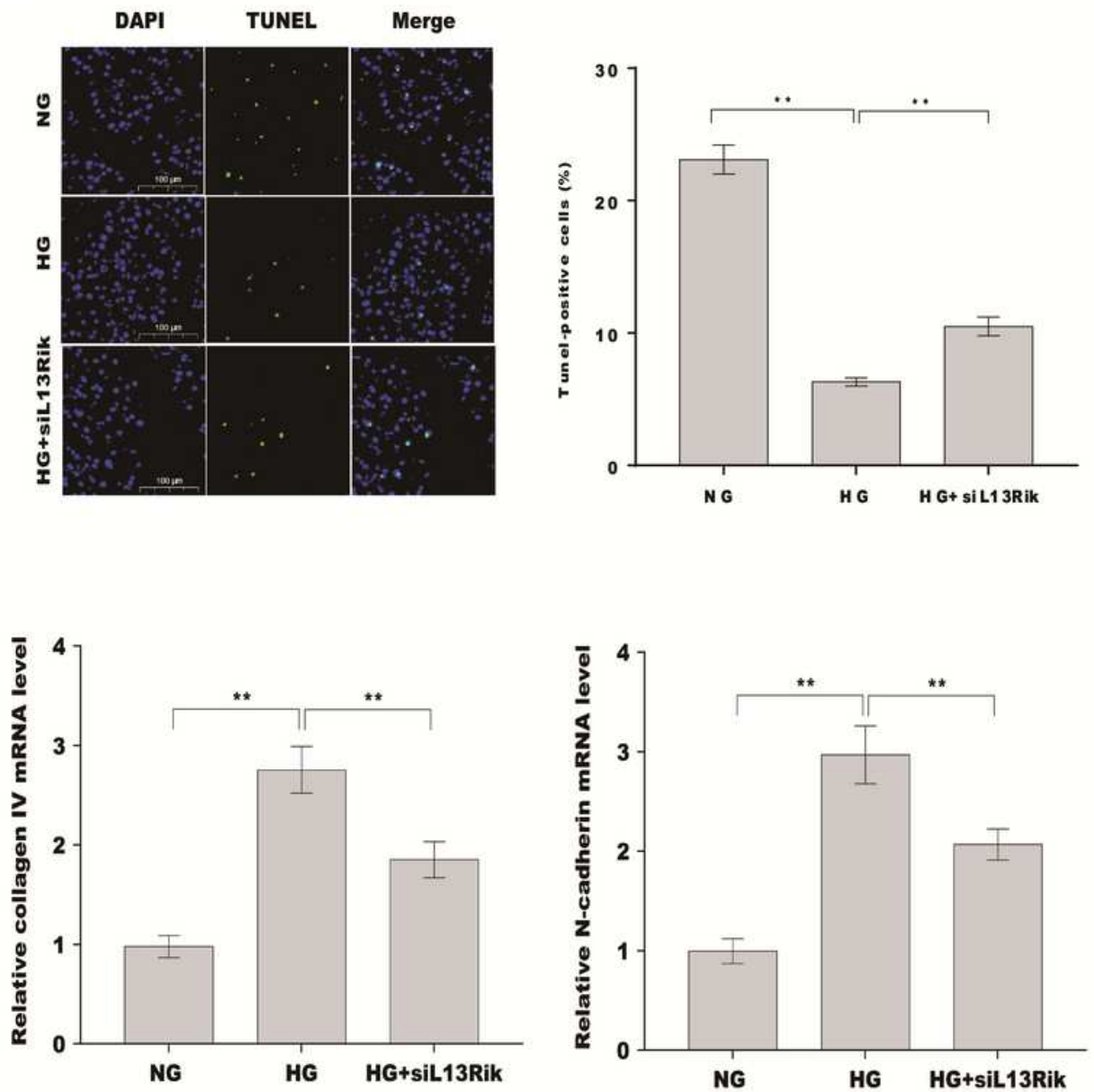

E

F

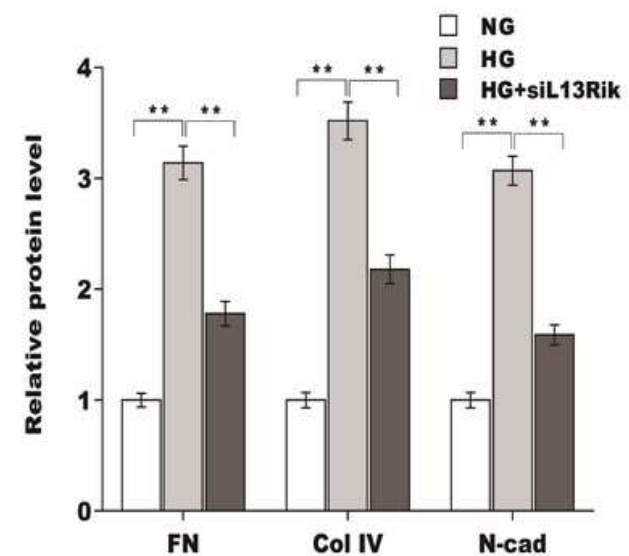

Figure 2

Knockdown of L13Rik suppressed HG-induced proliferation, ECM accumulation, and hypertrophy (A) Mesangial cell proliferation was assayed using CCK-8 after HG treatment with or without L13Rik knockdown. (B) Mesangial cell apoptosis was assayed using Tunel staining after HG treatment with or without L13Rik knockdown. (C) The mRNA levels of fibronectin (FN), collagen IV (Col-IV) and N-cadherin (N-cad) in mesangial cells after HG treatment with or without L13Rik knockdown were assessed by RTPCR. (D and E) The protein levels of FN, Col-IV and N-cad in mesangial cells after HG treatment with or 
without L13Rik knockdown were assessed by western blotting. $(F)$ The mesangial cell hypertrophy was assessed using total protein level per cell. The results show data from three independent experiments, expressed as the mean \pm SD. ${ }^{*} p<0.05$. ${ }^{* *} p<0.01$.

A

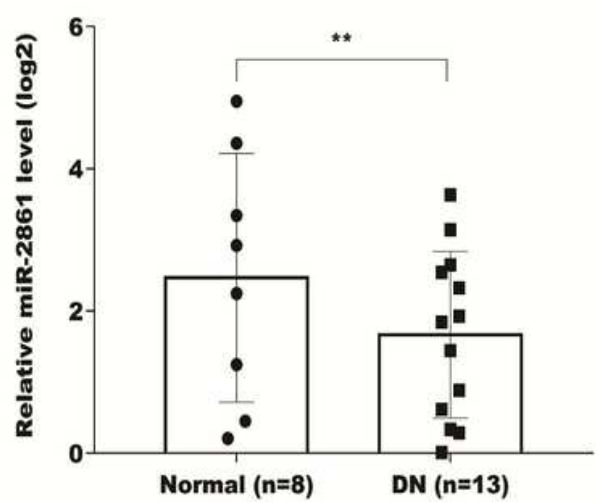

D

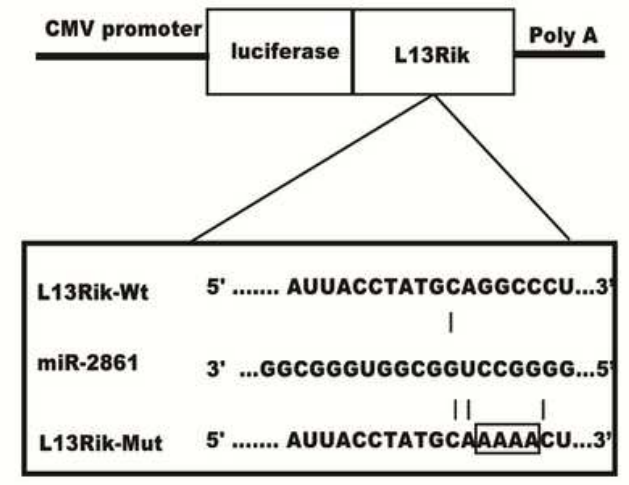

$\mathrm{F}$

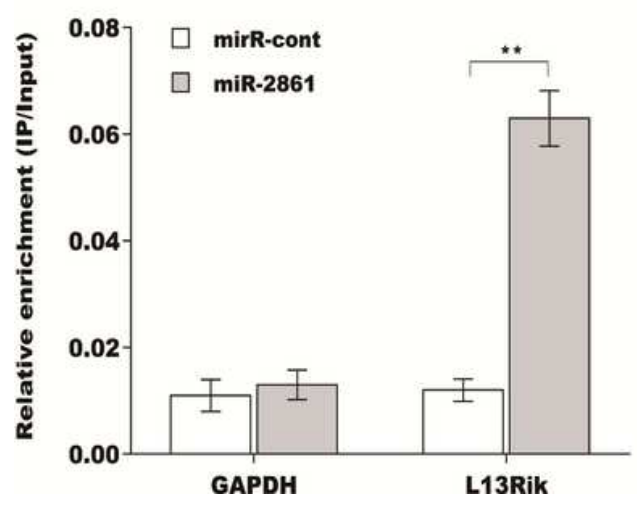

B
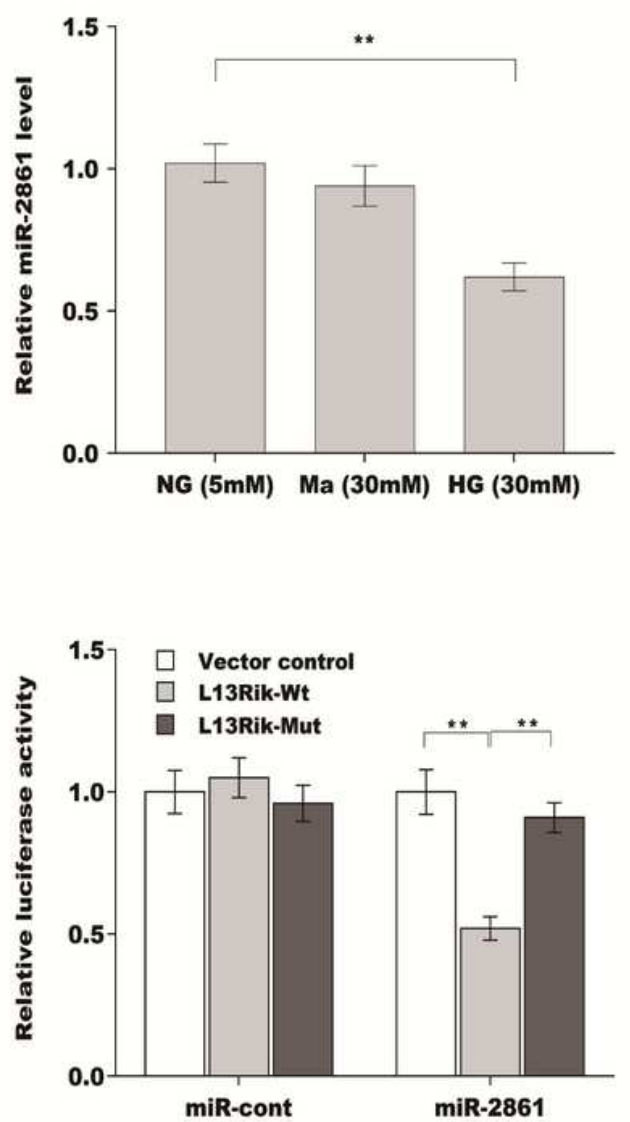

G

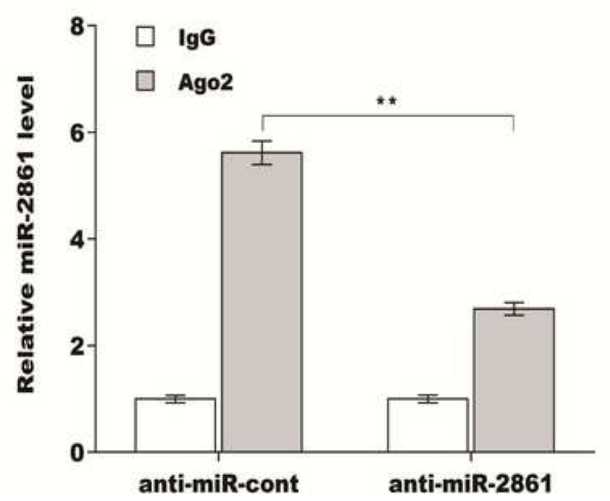

C

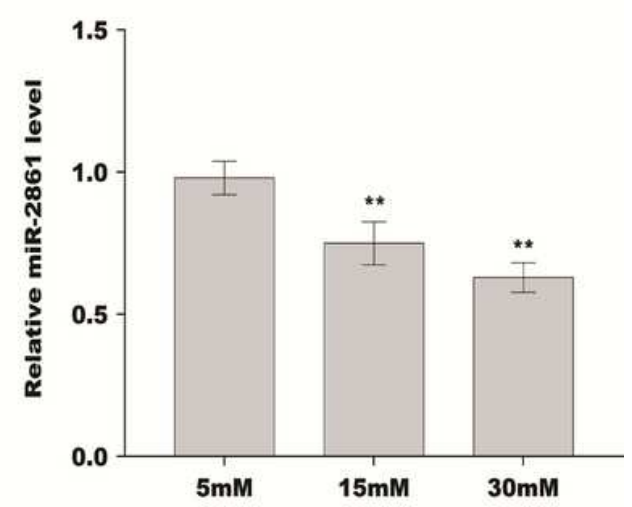

$\mathrm{E}$

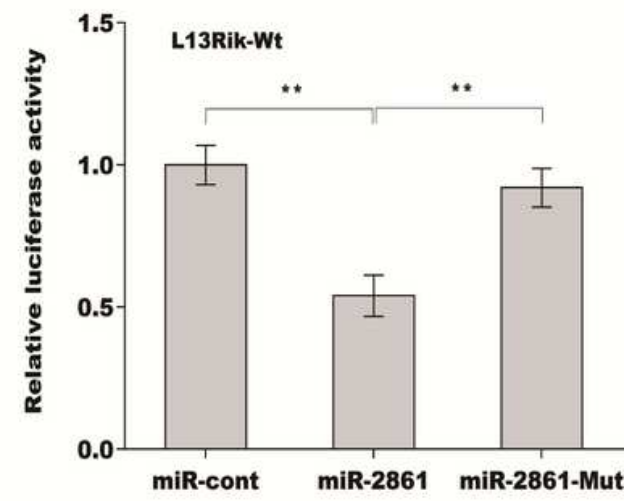

H

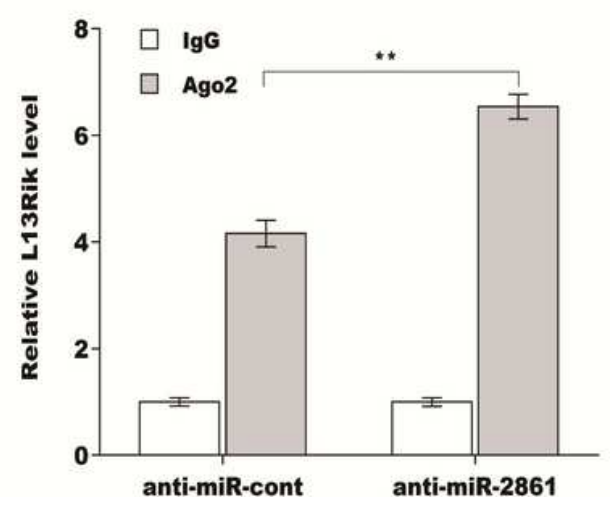

Figure 3

\section{L13Rik sponged miR-2861 by acting as ceRNA}

(A-C) qRT-PCR analysis of miR-2861 expression in 13 DN patients and 8 healthy controls (A), in diabetic rats (B), and in HG-cultured mesangial cells (C). (D) Schematic diagram of the miR-2861 site in L13Rik-wt- 
3'UTR and L13Rik-mutant-3'UTR. Luciferase activity was assayed in HEK293T cells co-transfected with miR-2861 and luciferase reporters containing L13Rik-wt-3'UTR and L13Rik-mutant-3'UTR. Data is presented as the relative ratio of firefly luciferase activity to renilla luciferase activity. (E) Luciferase activity was assayed in HEK293T cells co-transfected with L13Rik-wt-3'UTR with miR-2861-wt or miR2861-Mut. Data is presented as the relative ratio of firefly luciferase activity to renilla luciferase activity. (F) The direct interaction of miR-2861 with L13Rik was assessed by RNA pull-down assay. (G-H) RNA immunoprecipitation (RIP) analyses with anti-Ago2 antibody were carried out to assess the enrichment of L13Rik and miR-2861. The results show data from three independent experiments, expressed as the mean \pm SD. ${ }^{*} p<0.05 .{ }^{*} \mathrm{p}<0.01$.

A

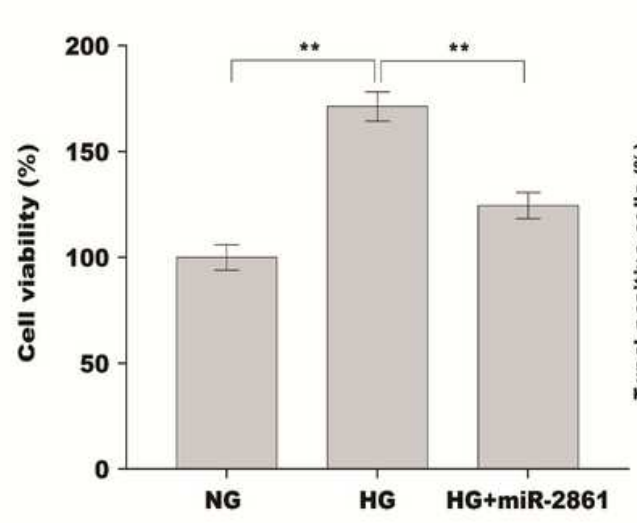

D

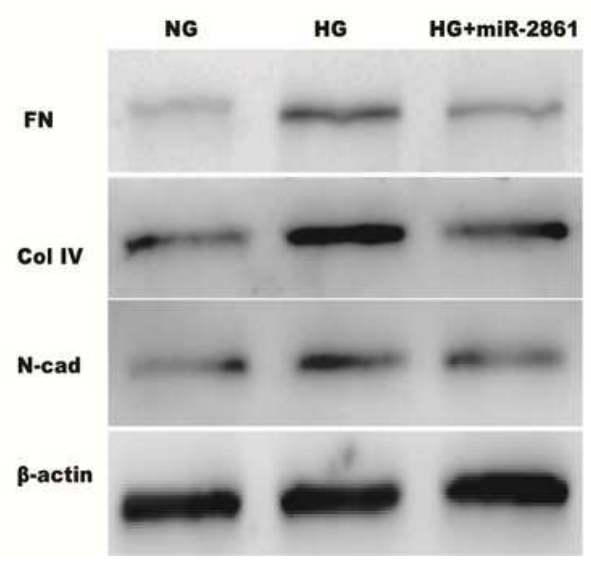

B

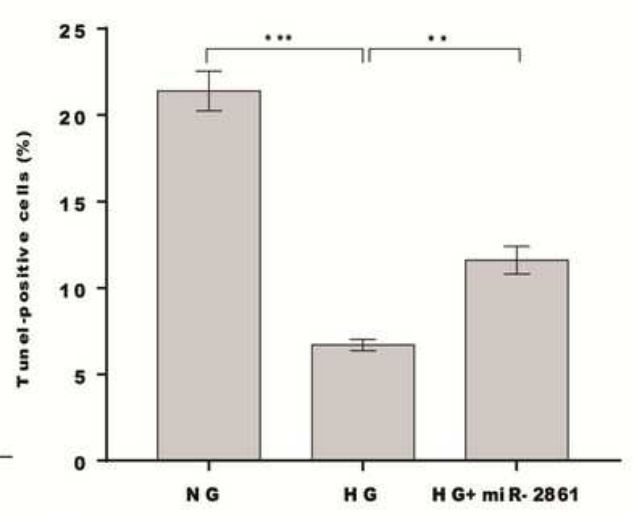

$E$

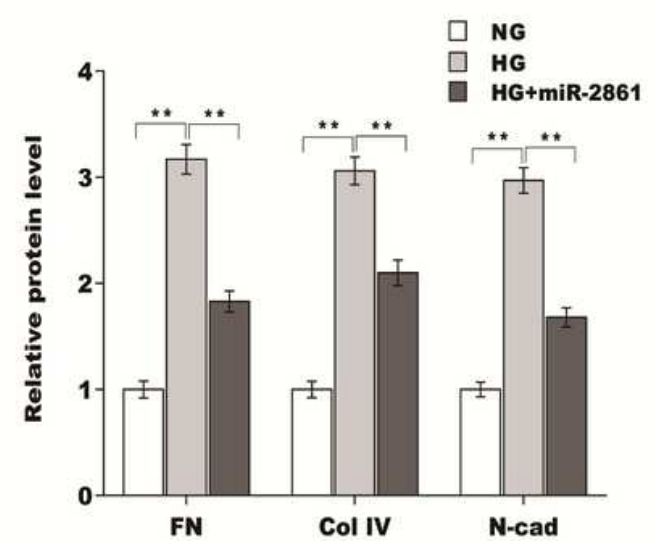

C

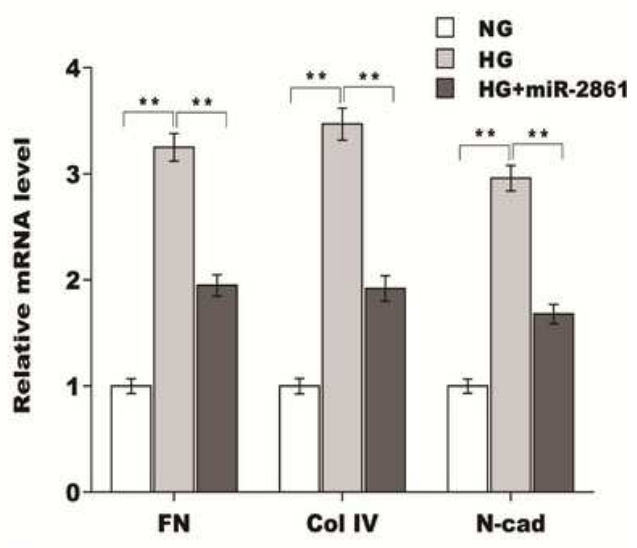

$\mathbf{F}$

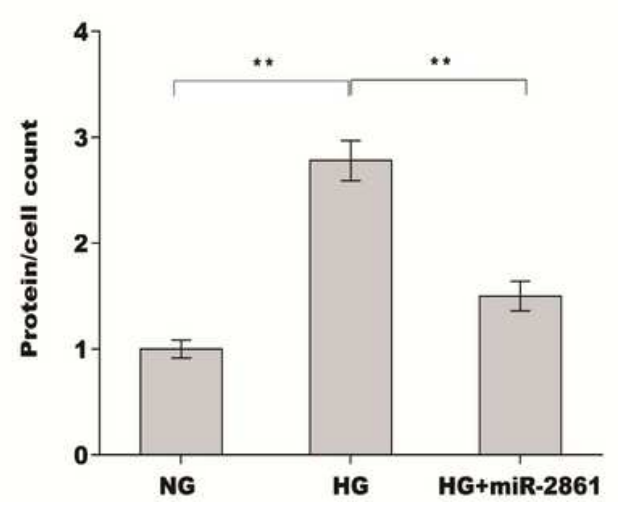

\section{Figure 4}

MiR-2861 suppressed HG-induced proliferation, ECM accumulation and hypertrophy (A) Mesangial cell proliferation was assayed using CCK-8 after HG treatment with or without miR-2861 overexpression. (B) Mesangial cell apoptosis was assayed using Tunel staining after HG treatment with or without miR-2861 overexpression. (C) The mRNA levels of FN, Col-IV and N-cad in mesangial cells after HG treatment with or without miR-2861 overexpression were assessed by RT-PCR. (D and E) The protein levels of FN, Col-IV and $\mathrm{N}$-cad in mesangial cells after $\mathrm{HG}$ treatment with or without miR-2861 overexpression were assessed by western blotting. $(\mathrm{F})$ The mesangial cell hypertrophy after HG treatment with or without miR-2861 
overexpression was assessed using total protein level per cell. The results show data from three independent experiments, expressed as the mean $\pm S D .{ }^{*} p<0.05 .{ }^{*} p<0.01$.

A

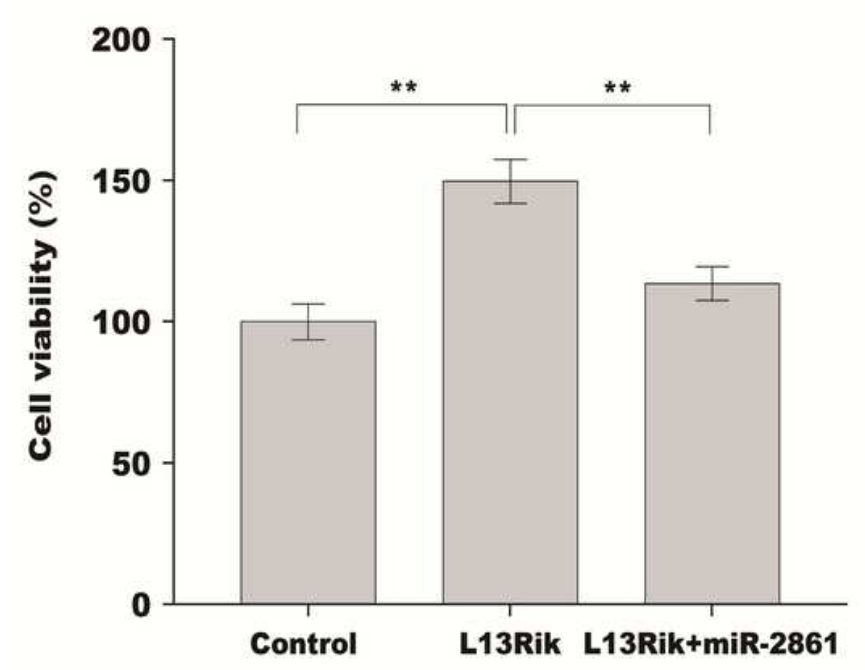

C

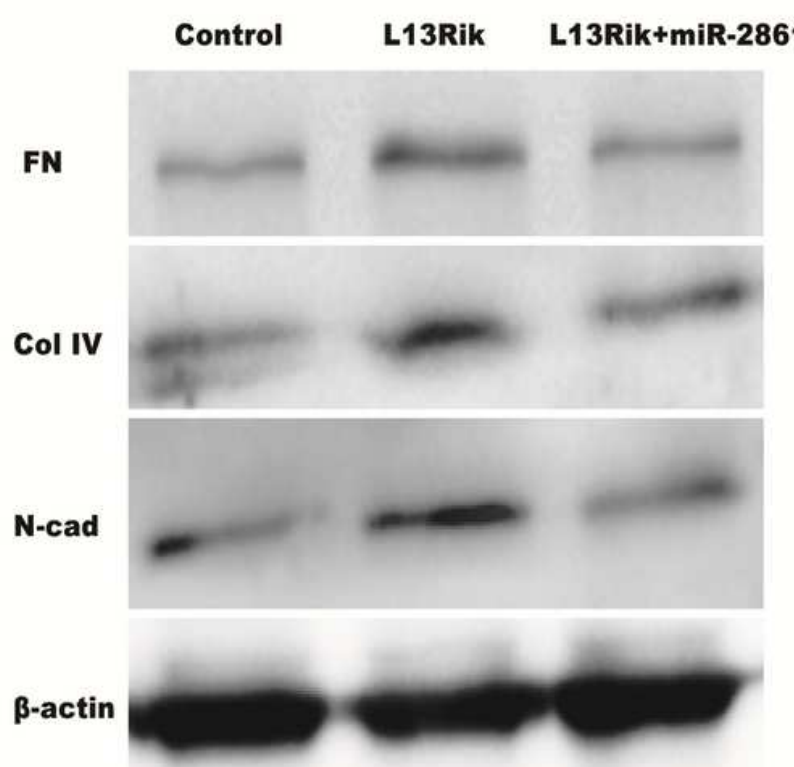

B

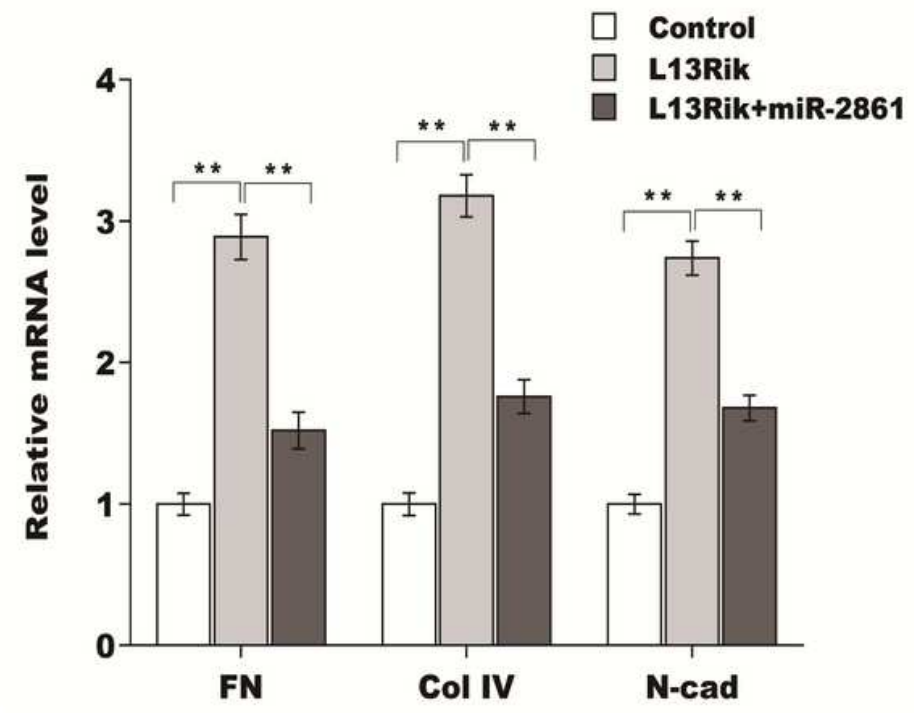

D

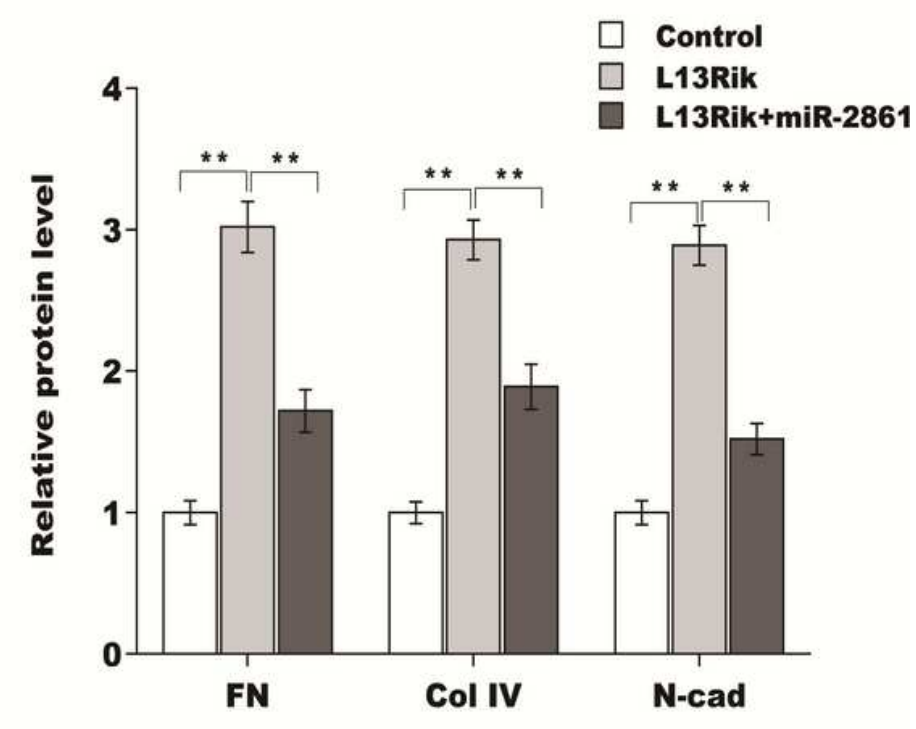

Figure 5

MiR-2861 antagonized the effect of L13Rik on hypertrophy (A) Mesangial cell proliferation was assayed using CCK-8 after overexpression with L13Rik in the presence or absence of miR-2861. (B) The mRNA levels of FN, Col-IV, and N-cad in mesangial cells after overexpression with L13Rik in the presence or absence of miR-2861 were assessed by RT-PCR. ( $C$ and D) The protein levels of FN, Col-IV and N-cad in mesangial cells after overexpression with L13Rik in the presence or absence of miR-2861were assessed by western blotting. The results show data from three independent experiments, expressed as the mean \pm SD. ${ }^{*} \mathrm{p}<0.01$. 
A

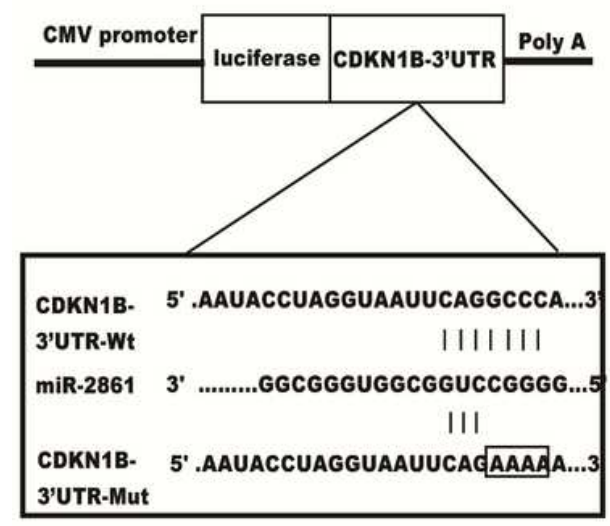

D

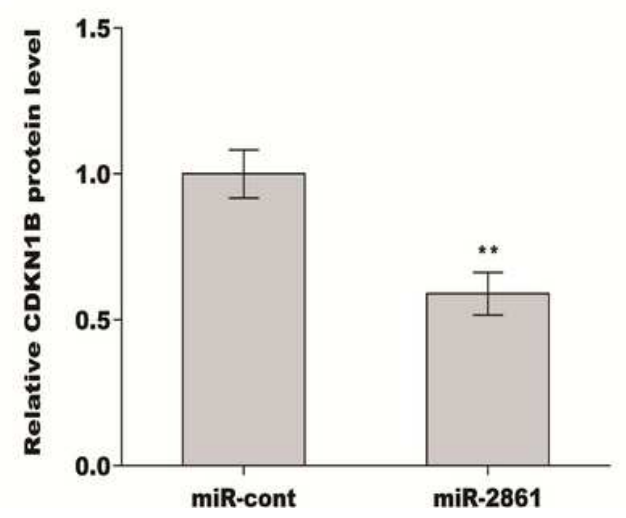

B

C

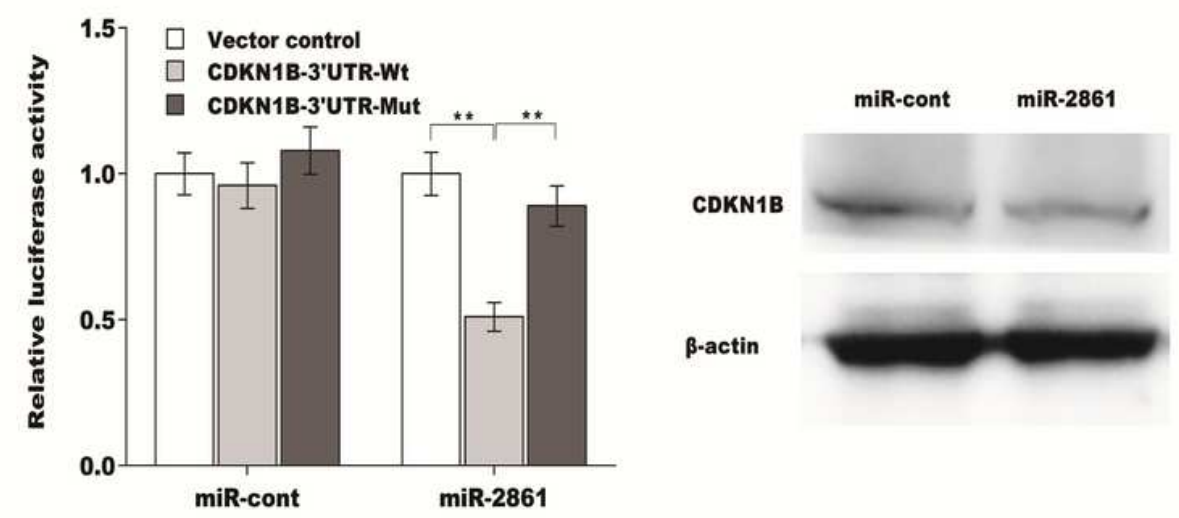

E

$\mathrm{F}$
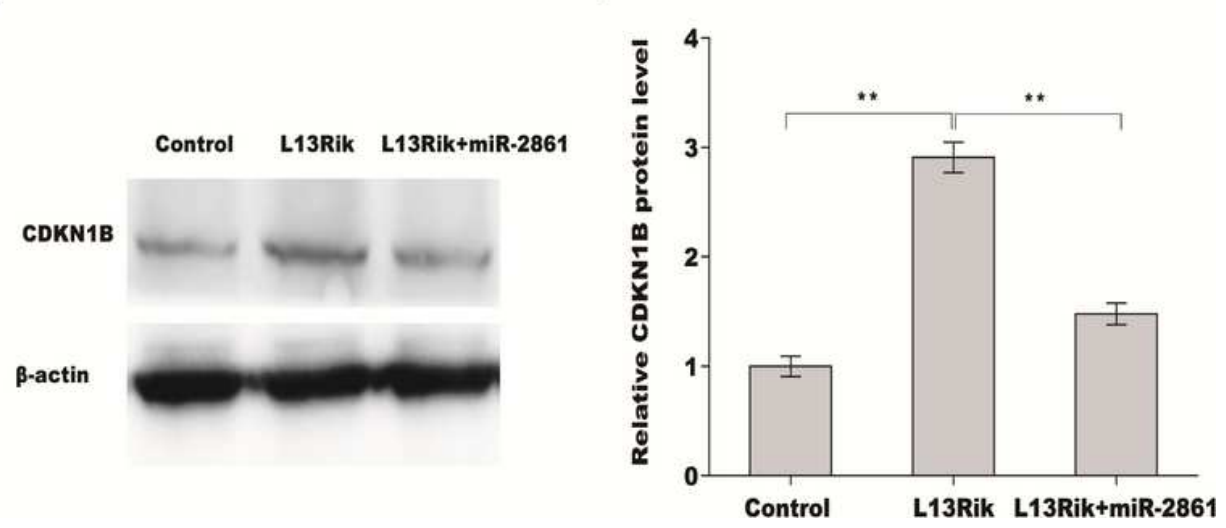

Figure 6

L13Rik promoted cell hypertrophy by modulating miR-2861/CDKN1B axis (A) Schematic diagram of the miR-2861 site in CDKN1B-3'UTR. (B) The activation of Luciferase was analyzed in HEK293T cells cotransfected with miR-2861 and luciferase reporters containing CDKN1B-3'UTR-Wt or CDKN1B-3'UTR-Mut. (C and D) Western blot analysis of CDKN1B expression in mesangial cells after miR-2861 overexpression. (E and F) Western blot analysis for CDKN1B protein level in mesangial cells after L13Rik overexpression in the presence or absence of miR-2861. The results show data from three independent experiments, expressed as the mean \pm SD. ${ }^{*} p<0.05 .{ }^{*} \mathrm{p}<0.01$.

\section{Supplementary Files}

This is a list of supplementary files associated with this preprint. Click to download.

- Table1.pdf

- SupportingTables1.pdf

- SupportingTableS2potentialmiRNAsspongedbyL13Rik.pdf

- SupportingTableS3dysregulatedmiRNAsinDNstudies.pdf

- SupportingfigS1.tif 
- SupportingfigS2.tif 